\title{
A Patient-Oriented Approach to Acne, Rosacea, Perioral Dermatitis, and Isotretinoin with Working Patient Informational/Instructional Sheets and a Review of Pathophysiology and Mechanisms of Therapeutic Agents
}

\author{
Craig G. Burkhart* \\ University of Toledo College of Medicine, 5600 Monroe Street, Suite 106B, Sylvania, Ohio 43560, USA
}

\begin{abstract}
Patient informational/instructional sheets can be used not only to assist in providing patients with baseline material regarding their condition, but also aid in physicians communicating a treatment protocol. Educational material regarding acne, adult acne, perioral dermatitis, and isotretinoin is included for perusal and discussion. Within this framework, one learns the unifying concept of the Propionibacterium acnes biofilm, methods to maximize production of benzoyl peroxide radicals (the active biological form of this compound), unplugging ability of oral antibiotics, and low-dose isotretinoin therapeutics.
\end{abstract}

Caring for patients involves a number of critically important elements including being sympathetic, understanding, supportive, and educating them about their condition and their treatment regime. Regarding that latter point, there are diverse methods that can be utilized to convey to patients information concerning their individual skin condition and therapy. One method would be patient informational sheets to assist in providing patients with baseline material regarding their condition and treatment. Of course, they should be written in language which most patients will find easy to comprehend, and offered after greeting the patient and performing a history and physical examination. In the case of acne and related conditions, it is also helpful to introduce the topic by using diagrams explaining that bacteria within the hair follicle unit produce toxins that cause inflammation, and that treatments will be directed at these bacteria, or their food source, the sebaceous gland.

Without further ado, figures 1 through 5 are the initial instructional sheets used in Sylvania, Ohio for the following conditions: acne, adult acne, perioral dermatitis, and isotretinoin. Please note there is an additional sheet for females who opt for accutane, due to all the additional steps required to obtain this drug for this sex. Of note, these informational sheets selected for discussion are for patients who either have no insurance or insurance which provide limited drug coverage. Certainly, with patients with standard insurances, trade name products become more economically feasible and informational sheets would be easily modified to reflect one's preferred therapies with this expanded therapeutic armamentarium. Physician-written instructional sheets parallel the clinical teachings and beliefs of the individual disease state. Thus, the remainder of this paper will discuss the material contained within these information sheets, and offering justification of the beliefs expressed within them.

*Address correspondence to this author at University of Toledo College of Medicine, 5600 Monroe Street, Suite 106B, Sylvania, Ohio 43560, USA; E-mail: cgbakb@aol.com

\section{ACNE BIOFILM: THE CENTRAL ISSUE IN ACNE PATHOGENESIS}

Beginning with the acne informational sheet, most textbooks suggest that the pathophysiology of acne vulgaris is an interplay of follicular hyperkeratinization, bacteria in the follicular canal, and sebum production. Targeting the microbiology of acne, $P$. acnes and its numerous immunologic ramifications constitute a major thrust of present research in the elucidation of the pathogenesis of inflammatory acne. Regardless of therapeutic modalities, $P$. acnes are always found harbored within the pilo-sebaceous unit, and the number of organisms does not necessarily correlate with clinical results. On point, antibiotic resistance in standard bacterial cultures is no longer a reliable assessment of therapeutic outcome [1]. Indeed there is growing evidence that alteration of the physical, biological, and chemical environment of the pilosebaceous unit is the primary function of all acne therapy [2]. The reason for this is found by applying the microbiological principle of biofilms to acne.

Expanding on this important concept, most bacteria exist not as free-floating microorganisms, but as biofilms. This designation means that populations of bacteria adhere to environmental surfaces (such as the pilosebaceous lining), and encase themselves in an extracellular polysaccharide which they secrete. The extracellular matrix usually comprises twothirds of the biofilm mass and acts as a protective exoskeleton and physical barrier, limiting the effective antimicrobial concentrations within the biofilm microenvironment. In the case of acne, the $P$. acnes biofilm allows the organism to survive in hostile environments [1-3]. The complete $P$. acnes genome supports the existence of the $P$. acnes biofilm [4]. In short, the $P$. acnes genome contains clusters of genes involved in polysaccharide capsule biosynthesis of a glycocalyx polymer, which account for adherence to a surface in biofilm formation secreted by the organisms [5]. For example, there are several sequences granting the genomic machinery necessary to make biofilm, such as UDP-Nacetylglucosamine 2 epimerase and glycosyl transferases [5]. Indeed, these substances assisting adherence of the bacteria are likely the incriminating cause of comedones as well. 
$P$. acnes is the overwhelmingly predominant microorganism in the pilosebaceous unit. It is serologically and biochemically identical to Corynbacteriaum parvum, a potent stimulator of the reticuloendothelial system. It is an indigenous bacteria which has been proven to be pathogenic in several cutaneous disease states including granulomas in sarcoidosis [6]. Moreover, incriminating studies such as API 20A systems, 16sRNA sequencing, DNA-DNA hybridization, XTT reduction method, and scanning electron microscopy have suggested that the $P$. acnes biofilm is the inciting factor for orthopedic infections [7] and in fatal bacterial granulomas after trauma $[8,9]$.

Standard laboratory in vitro bacterial sensitivity studies with $P$. acnes from fatal bacterial granulomas did not prove clinically effective in vivo given the increased antibacterial resistance from biofilm formation. However, the $P$. acnes biofilm can be formed in vitro, in which it takes 96 hours for maturation of the biofilm in culture. Of significance from such studies are that certain antibiotics, such as lincomycin, in sub-minimal inhibitory concentrations, enhances and accelerates the biofilm forming ability of the P. acnes. Thus, long-term therapy with certain antibiotics can improve biofilm formation and clinically aggravate the condition. Of note, the minimum inhibitory concentration of $P$. acnes was not affected by long term therapy with minocycline, one of the common antibiotics used in the treatment of acne. Such studies have strong implications in acne therapeutics as well. On passing, testing to develop methods to decrease adhesion of the bacteria to surfaces and the subsequent biofilm formation are warranted, and in vitro $P$. acnes biofilm studies should be utilized in our acne research.

\section{BENZOYL PEROXIDE, WITH ALTERATIONS, AS THE PREFERRED TOPICAL TREATMENT FOR ACNE}

Any instructional sheet on acne will be biased as to which agents a particular physician feels makes the most significant alteration to the microenvironment in which $P$. acnes reside. In line with previous publications, these authors favor benzoyl peroxide as the initial topical agent for acne, but with some modifications from its normal usage.

Benzoyl peroxide is well absorbed into the epidermis and concentrated in the pilosebaceous units and definitely alters the $P$. acnes microenvironment. Benzoyl peroxide is antibacterial, functions as a peeling agent, has comedolytic activity, and reduces free fatty acid levels. Additionally, there are conflicting reports of sebosuppressive effects [10].

Concomitant topical treatment of benzoyl peroxide with certain topical antibiotics, namely clindamycin and erythromycin, has been shown to decrease the in vivo follicular counts of $P$. acnes and to be more clinically effective for acne than their individual components alone [11-14]. The clinical improvement is not, however, because clindamycin and/or erythromycin are antibacterial, because antibacterial resistance to these agents are reported to be as high as $94 \%$ in 2003 [15]. The reason for the combination giving improved therapeutics is that clindamycin and erythromycin are both chemically tertiary amines. This chemical structure acts as a stimulant for the production of benzoyl peroxide radicals. The joint treatment of benzoyl peroxide and a tertiary amine also greatly outperforms tretinoin with erythromycin, with clinical differences appearing within 2 weeks of usage [16]. Moreover, a non-antibiotic chemical with a tertiary amine, such as an allylamine, can be used in concert with benzoyl peroxide and also provide similar therapeutic responses [17].

In other words, a basic tenet is that the most active biological and chemical form of benzoyl peroxide is its radical state. Examples in industry include benzoyl peroxide radicals initiating a process called polymerization that is used as human bone cement and for mending cracks on wings of airplanes [17-21]. In medicine, in vitro studies have demonstrated that benzoyl peroxide radicals have increased antibacterial and antiyeast coverage [18]. Thus, to produce a more effective benzoyl peroxide, one wants to convert the chemical into its more active state, which is the benzoyl peroxide radical. This chemical transformation can be maximized by means of a stimulant, an accelerator, and/or modification of the vehicle.

As allude to above, the most common stimulant is the combining of benzoyl peroxide with a tertiary amine, which is a substance that has an accessible nitrogen molecule that is already partially saturated with carbon molecules. Examples of tertiary amines in our armamentarium include certain antibiotics (erythromycin and clindamycin), as well as antifungal agents which are allyalamines, such as terbenafine and butenafine. This tertiary amine readily converts benzoyl peroxide into its more active radical state by the transference of electrons.

The accelerator for this reaction between benzoyl peroxide and a tertiary amine are trace metals, such as zinc. From chemistry and industry, we know that accelerators reduce the energy level needed for the reaction between benzoyl peroxide and a tertiary amine to occur. Their presence would accentuate the production of radicals.

Independently of the former methods to improve benzoyl peroxide potency, there are a few ways by which one can merely alter the base to maximize the power of benzoyl peroxide. For example, benzoyl peroxide is more active when the base has high polyethylene glycol and low water content.

Significant clinically is that once formed, benzoyl peroxide radicals last for miniseconds. To maximize biological effects, one should mix the benzoyl peroxide with the tertiary amine on the skin surface to maximize results. Although there are various methods to improve production of benzoyl peroxide radicals, in patients with limited income, one inexpensive option is the use of a tertiary amine (such as erythromycin or clindamycin solution) applied to the skin surface, followed by benzoyl peroxide in the form of an inexpensive wash.

\section{ORAL ANTIBIOTICS IN ACNE}

To maximize patient improvement, systemic antibiotics are indicated in the treatment in acne because they offer excellent results. Textbooks still report that they affect the course of acne by causing a decrease in the population of $P$. acnes and thereby inhibiting bacterial lipases, even though reports of the incidence of resistant strains of $P$. acnes to clindamycin, tetracycline, erythromycin, and trimethoprimsulfamethoxazole have continued to increase [22, 23]. Again, antibiotics do not eliminate $P$. acnes, but rather control the 
$P$. acnes biofilm from producing toxins disadvantageous to the host. After good clinical results are achieved with oral antibiotics, most patients are able to reduce/titrate the amount to a level by which their $P$. acnes biofilm is controlled from secreting an overabundance of toxins.

There has been discussion concerning sub-antimicrobial dosing with antibiotics improving acne. Such doses do not kill bacteria and are stated that therefore they cannot induce resistance. However, this concept is better appreciated within the framework of the acne biofilm, in which different patients will find different levels of systemic antibiotics required to control the P. acnes biofilm.

In most practices, tetracycline and its derivatives such as minocycline and doxycycline are the preferred oral antibiotic. Different situations alter this schema. For example, female patients on oral contraception are usually given either oral erythromycin or trimethoprim-sulfamethoxazole, as the package insert does not have as stringent wording on possible interaction with birth control pills.

On passing, antibiotics also have unplugging activity. In short, $P$. acnes secretes biological glue allowing for adherence to follicular walls. This adhesive glue finds its way also into the sebum, leading to the binding of corneocytes in the infundibulum leading to comedone formation [24].

\section{ADULT ACNE VS ROSACEA}

Although rosacea has been recently defined as the presence of one or more of the following primary features (flushing, nontransient erythema, papules and pustules, telangiectasia) and one or more of the following secondary features (burning, plaque, dry appearance, edema, ocular manifestations, peripheral location, phymatous changes) [25], a more appropriate, and less confusing, terminology reverts to its original name of acne rosacea. This eliminates the confusion of pathogenesis because the acne biofilm again is the predominant factor. Because of this fundamental relationship between acne and adult acne, the informational sheets have similar verbiage.

As an aside, there is awareness that epidermal atrophy does occur in some individuals with rosacea, leading to visualization of the underlying dermal vessels (telangiectasias), but this is a separate entity. Nevertheless, by using appropriate antibiotic therapy, the livid red erythema seen in some adult acne patients does dissipate when those bacterial toxins which cause at least some of the vasodilatation are suppressed.

With age, the $P$. acnes biofilm changes as there are alterations in the hair follicle lining, P. acnes attachment, and in sebaceous gland production. Clinically there are some individuals in which features of adult acne and seborrheic dermatitis co-exist. In these individuals, ketoconazole cream to reduce the overgrowth of yeast elements is helpful. A discussion of these newer concepts regarding seborrheic dermatitis is available in a publication in The Open Dermatology Journal [26].

\section{PERIORAL DERMATITIS, THE ACNE THAT LOOKS A BIT DIFFERENT}

Perioral dermatitis is a relatively common inflammatory disorder of facial skin in which small papules and pustules occur around the mouth. The condition usually arises spontaneously over the course of several weeks. In the majority of cases, there is a striking dependency on topical corticosteroids.

Again, this condition represents an alteration of the acne biofilm. Again, the central treatment is restoring the balance within the hair follicle unit. Again, the informational sheet provided reflects this common thread among these acne conditions.

\section{ISOTRETINOIN AND THE iPLEDGE SYSTEM}

Isotretinoin has revolutionized the management of severe acne that is unresponsive to antibiotics. Being a derivative of Vitamin A, it shares almost identical side effects, but rarely do the side effects require interruption of therapy. The beauty of isotretinoin is the completeness and longevity of the remission.

Studies have shown, similar to vitamin A, that the side effects are basically dose related. Although textbooks continue to state that low dosages increase the incidence of relapses, several studies have shown that this has been exaggerated. For example, the majority of patients in Sylvania, Ohio are treated with $20 \mathrm{mg}$ of isotretinoin daily for the entire 5 months of therapy with excellent results.

The greatest concern during isotretinoin therapy is the risk of the drug being administered during pregnancy and thereby inducing teratogenic effects in the fetus. The iPledge System issued by the FDA constitutes a verification process requiring a set process for the usage of isotretinoin in both males and females taking the drug. As the booklets are cumbersome to read and represent to some extent, legal documents, informational sheets speed patient understanding and comprehension of the necessary points for patient/physician discussion.

\section{CONCLUSION}

A combination of topical and systemic agents can greatly reduce the amount and severity of acne in most cases. An understanding of the acne biofilm aids in patient understanding and in better appreciation of acne pathogenesis and in therapeutic avenues. Patient informational/instructional sheets can be used not only to assist in providing patients with baseline material regarding their condition, but also aid in physicians communicating a treatment protocol. By seeing how colleagues approach various disease entities, hopefully there will be further discussion and continuation in the improvement of patient education and clinical response with acne, adult acne, perioral dermatitis, and accutane.

\section{Patient Information on Acne}

\author{
Craig G. Burkhart, MPH, MD \\ 5600 Monroe Street, Suite 106B, Sylvania, Ohio \\ 43560, USA
}

Acne is a skin disease that is common in adolescents, but can occur in all ages. The main cause of acne is bacteria that live within the pores and hair follicle units, under- 
neath the skin surface. These bacteria produce several toxins that cause inflammation. These bacteria produce a shield from the body's natural immune system (called a biofilm), and one cannot totally eliminate the bacteria by means of antibiotics. Both oral and topical treatment is directed at altering the environment in which these bacteria live, so they do not make these bad toxins. This is why extended courses of oral antibiotics and topical agents are often required for good control.

\section{Common Concerns Related to Acne}

Acne does eventually go away by itself, but there is no way to tell when it will stop even with medical treatments. Mother Nature has different end points for different people.

If one knows of some food that makes one's acne worse, avoid that food. However, there is no scientific evidence that elimination of any specific food (such as nuts, chocolate, or dairy) from the diet makes any difference in the course of the disease.

In terms of make-ups for females with acne, use water-based or oil-free products.

Acne is not caused by not washing one's face. In fact, patients with acne tend to wash their face more than people without acne. I usually suggest a specific prescription cleanser for my acne patients (if your insurance pays the majority of the price for the product), as this cleanser should augment some of our topical remedies.

If one has a tendency to squeeze blackheads, use gentle pressure. If the plug doesn't come with mild pressure, give up and continue the topical unplugging agents longer. Forcing the blackhead out with excessive pressure can cause damage below the surface, more likely resulting in a scar.

Acne can cause scaring. Our medical treatment hopefully will eliminate any further scarring. Only after medical treatment has quieted the acne down, some patients might consider simple, non-surgical (topical) peels from my office. Others may require surgical dermabrasion in an operative setting, a procedure performed by plastic surgeons. No insurance plan allows me to refer you to anyone who performs these procedures. Thus, one would have to have your primary physician refer you to plastics, or assume that such a procedure will be outside your insurance coverage.

The standard initial treatment of acne includes a topical solution to be applied in the morning, an acne wash which can be used once or twice daily, and oral antibiotics. Patients return in 6 to 8 weeks after the initial treatment to see what alterations have to be made. Often, six months of treatment is often needed to see the full effects of a specific oral antibiotic therapy.

I allow pharmacists to substitute similar (sister) products if more cost effective for you. For example, minocycline can be substituted for doxycycline. Of note, some pharmacists put a sticker to avoid the sun on one of the popular antibiotics for acne, namely minocycline; this can be disregarded as this drug only makes one $1 \%$ more sensitive to the sun. Unlike some pharmacists, I also recommend taking minocycline with meals, as the absorption is very minimally affected by food. If you check the package insert, $89 \%$ of minocycline is absorbed with meals, which is almost the same as the absorption when taken in between meals. With doxycycline, it is photosensitizing and should be taken with dinner to reduce this side effect.

FYI: If more cost effective for you, the (1) antibiotic pills, (2) topical acne solution, and (3) topical wash prescribed in Dr. B's initial treatment for acne, are each obtainable by means of the $\$ 4$ prescription plan at several retailers such as Wal-Mart and Pharm. That's $\$ 4 /$ month for each script. (Please note that depending upon which oral drug prescribed, Wal-Mart or Pharm will give you between a 20 to 30 day supply for that price). You will want to compare that price with that obtained via your insurance plan.

\section{Patient Information on Adult Acne (The Acne form of Rosacea)}

\author{
Craig G. Burkhart, MPH, MD,
}

\section{Monroe Street, Sylvania, Ohio, USA}

Adult acne is a common, chronic skin disease that causes inflammation and redness of the face. The main cause is bacteria that live within the pores and hair follicle units, underneath the skin surface. These bacteria produce several toxins that cause inflammation. These bacteria produce a shield from the body's natural immune system (called a biofilm), and one cannot totally eliminate the bacteria by means of antibiotics. Treatment is directed at controlling these bacteria by altering the environment of the hair follicle unit in which they live. This is why extended courses of oral antibiotics and topical agents are required for good control.

\section{Common Concerns}

- $\quad$ Adult acne is not a serious medical problem. It is not associated with any other disease states.

Adult acne does not usually go away by itself. Although it responds extremely well to treatment, the disease usually requires maintenance therapy to keep it from returning.

Hot beverages, alcohol, and spicy foods tend to worsen the condition. However, oral treatment for adult acne is so good that food restrictions are unnecessary.

Adult acne is not caused by not washing one's face. Don't over wash your face.

In men, there is the rare form of adult acne (called rhinophyma) in which the oil glands greatly enlarge causing the nose to grow in size. This can often be controlled by medical treatments. 
- $\quad$ Some patients have an associated blepharitis, which are red, dry eyes which respond nicely to oral antibiotics discussed below.

Some patients with adult acne also have skin atrophy (thinning of the first layer of the skin so that one can see the tiny blood vessels under the skin surface with the naked eye). This is a distinct problem, but oral treatment may assist this condition.

Some patients with adult acne also have an associated dryness of the nasal-labial region and nasal brow. This associated problem is seborrheic dermatitis. When this is present, I will adjust the topical therapy to address this added skin problem. With seborrheic dermatitis, one doesn't make good enough protective oils to control fungus and bacteria on the skin, thereby requiring a topical agent to address the fungus, and an oral agent to attack the bacterial component.

The standard initial treatment of adult acne includes a topical solution to be applied in the morning, an acne wash which can be used once or twice daily, and oral antibiotics. Patients return in 6 to 8 weeks after the initial treatment to see what alterations have to be made. There is usually an $80 \%$ improvement of severe adult acne in 2 months after starting therapy. Once one achieves $90 \%$ improvement (let's guess that this occurs in 3 months of treatment), then most patients can lower the amount of oral antibiotic that is needed to keep the condition away. For example, while some patients require 2 tablets days of antibiotic, others require only 1 tablet every other week. Thus, you will have to adjust the dose of the antibiotic to your needs.

I allow pharmacists to substitute similar (sister) products if more cost effective for you. For example, minocycline can be substituted for doxycycline. Of note, some pharmacists put a sticker to avoid the sun on one of the popular antibiotics for acne, namely minocycline; this can be disregarded as this drug only makes one $1 \%$ more sensitive to the sun. Unlike some pharmacists, I also recommend taking minocycline with meals, as the absorption is very minimally affected by food. If you check the package insert, $89 \%$ of minocycline is absorbed with meals, which is almost the same as the absorption when taken in between meals. With doxycycline, it is photosensitizing and should be taken with dinner to reduce this side effect.

FYI: If more cost effective for you, the (1) antibiotic pills, (2) topical acne solution, and (3) topical wash prescribed in Dr. B's initial treatment for acne, are each obtainable by means of the $\$ 4$ prescription plan at several retailers such as Wal-Mart and Pharm. That's $\$ 4 /$ month for each script. (Please note that depending upon which oral drug prescribed, Wal-Mart or Pharm will give you between a 20 to 30 day supply for that price). You will want to compare that price with that obtained via your insurance plan.

\section{Patient Information on Perioral Dermatitis}

\author{
Craig G. Burkhart, MPH, MD \\ 5600 Monroe Street, Suite 106B, Sylvania, Ohio \\ 43560, USA
}

Perioral dermatitis is a facial condition that looks often like a rash, but is a form of acne. Thus, one might have small pink papules, pustules, patches with fine scale, and redness on the chin and checks that is concentrated somewhat around the mouth. This inflammation and redness of the face can also be associated with some itching. Like acne, the main cause of perioral dermatitis is a specific bacteria that lives within the hair follicle unit, underneath the skin surface. These bacteria are able to produce several toxins that cause inflammation. Of note, these bacteria produce a shield from the body's natural immune system (called a biofilm), and one cannot eliminate the bacteria by means of antibiotics. Thus, treatment is directed at controlling these bacteria by altering the environment of the hair follicle unit in which they live. This is why extended courses of oral antibiotics and topical agents are required for good control.

\section{Common Concerns}

Perioral dermatitis is not a serious medical problem. It is not associated with any other disease states.

Perioral dermatitis does not usually go away by itself. Although perioral dermatitis responds extremely well to treatment, the disease often requires maintenance therapy for up to 6 months to keep it from returning.

Hot beverages, alcohol, and spicy foods tend to worsen the condition. However, treatment for perioral dermatitis is so good that food restrictions are unnecessary.

Perioral dermatitis is not caused by not washing one's face. Once on treatment, you can wash one's face with whatever product you wish.

Perioral dermatitis rarely causes any facial scaring.

Some patients with perioral dermatitis have used topical steroids on the skin to reduce the redness and itching. Topical steroids, however, allow the bacteria that cause the problem, to grow in numbers. It is very important to stop the topical steroids. This could easily cause the skin to get redder for a few days (because steroids make blood vessels shrink and eliminating the steroids will cause 
the vessels to dilate), but one has to suffer a few days of flaring to correct the problem. If female, you can use extra foundation during this interval of time to hide the redness.

The standard initial treatment of perioral dermatitis includes a topical solution to be applied in the morning, and oral antibiotics. Patients return in 6 to 8 weeks after the initial treatment to see what alterations have to be made. There is usually an $80 \%$ improvement in 2 months after starting therapy. Once one achieves $90 \%$ improvement, then most patients can lower the dose or stop oral antibiotics. Now, if you find the prescription solution irritating at first, use whatever moisturizer feels good to your skin for the first two weeks.

I allow pharmacists to substitute similar (sister) products if more cost effective for you. For example, minocycline can be substituted for doxycycline. Of note, some pharmacists put a sticker to avoid the sun on one of the popular antibiotics for acne, namely minocycline; this can be disregarded as this drug only makes one $1 \%$ more sensitive to the sun. Unlike some pharmacists, I also recommend taking minocycline with meals, as the absorption is very minimally affected by food. If you check the package insert, $89 \%$ of minocycline is absorbed with meals, which is almost the same as the absorption when taken in between meals. With doxycycline, it is photosensitizing and should be taken with dinner to reduce this side effect.

FYI: If more cost effective for you, the (1) antibiotic pills, and (2) topical acne solution, prescribed in Dr. B's initial treatment, are each obtainable by means of the $\$ 4$ prescription plan at several retailers such as Wal-Mart and Pharm. That's \$4/month for each script. (Please note that depending upon which oral drug prescribed, Wal-Mart or Pharm will give you between a 20 to 30 day supply for that price). You will want to compare that price with that obtained via your insurance plan.

\section{Patient Information on Isotretinoin (Trade Names Being Accutane, Claravis, Amnesteem and Sotret)}

\author{
Craig G. Burkhart, MPH, MD
}

5600 Monroe Street, Suite 106B, Sylvania, Ohio 43560, USA

Accutane is a Vitamin A derivative that has been available since September 1982 for acne. It is the most effective drug available for acne with most patients experiencing $60 \%$ improvement in 2 months, and over $90 \%$ in 5 months. Of note, results vary and some patients are not significantly improved until the fourth month of treatment. Because a son of a congressman from Michigan had depression while on accutane and because in high doses accutane can cause birth defects (as can Vitamin A in high doses), there is much more paperwork than normal with this drug.
A single five-month course of accutane usually results in almost complete clearance of acne in most patients. Moreover, if one is over 20 years old, acne normally does not return. If under 20 years old, acne may return after a full treatment (but not to the severity of its earlier state). Many dermatologists consider low dose accutane safer than oral antibiotics.

There are some concerns with accutane:

Any female who is pregnant, or conceivably could become pregnant while taking accutane, must not receive this drug. Accutane can cause devastating effects on a fetus conceived while the mother is on accutane. I use accutane with fertile females only if they follow the required guidelines, which includes use of two forms of birth control, extra blood tests, and an additional form filled out by one's OB/GYN physician.

One cannot take accutane for prolonged periods of time because joint problems could develop. That is why the duration of treatments is only five months. If under 20 years old, I usually recommend taking accutane for five months only once a year to avoid such problems. (In such cases, I would use other therapies the remainder of the year if needed.)

Depression has been recorded in patients using high doses of accutane, including a congressman's son (who was also on street drugs) that has lead to the accompanying informed consent/patient agreement. I always use low dosages of the drug that, by several studies, achieves equivalent results (to high dose) with less concern for side effects (i.e. Journal of the American Academy of Dermatology 2006;54:644-6).

While on accutane, do not take any supplemental Vitamin A. (Vitamins B and C are fine to take.) Also, do not take tetracycline and/or minocycline in conjunction with accutane.

The only sport that I recommend not taking accutane with is wrestling, because nosebleeds can more easily occur given the nature of the sport. Wrestlers take accutane during the off-season.

Two blood tests of cholesterol and triglyceride are required while on accutane during the first and second months of therapy. Each blood drawing should be performed after a 12-hour fast (i.e. take before breakfast after not eating after dinner the night before). You may have only water during the fast. You also have to select a lab to draw your blood that is covered by your medical insurance. If unsure as to where you should have your blood drawn, you will have to call your insurance company.

Unless problems arise, I will only need to see you monthly during the treatment. Thereafter, I only need to see you if significant acne remains, or if the acne returns at a later time.

One side effect from accutane that is almost always seen is dry lips. Frequent use of Chap Stick and avoiding wetting the lips with saliva will help. Dry skin can occur and you may need extra moisturizers on the skin. I do not 
advise using any topical drying acne skin products while on accutane. The nasal mucosa can also dry and crack causing a minor nosebleed. Humidifiers may be helpful if this occurs. Occasionally patients require artificial tears due to dryness of the eyes. Rare side effects include muscle aches, peeling of the palms and soles, headaches, and night blindness. If any serious problems arise, please notify me.

The FDA has established the iPledgeProgram to oversee the usage of this drug. When Dr. Burkhart signs you into the program, you will get an I.D. iPledgeProgram Card which you need each time you go to the pharmacy for a refill prescription. Do not lose this I.D. Card. Also, mail your I.D. Card back to us with your name taped to it, at the completion of your treatment (where we will keep it in your chart).

One must purchase accutane within 7 days of obtaining the prescription, or it is voided. The iPledge system requires a monthly return visit.

NOTE: My office needs to register you over the internet into the iPledge computer system before you go to the pharmacy. This process may take 20 minutes to complete. If you have problems with iPledge, their toll-free phone number is 1-866-495-0654 and their website is www.ipledgeprogram.com.

\section{Patient Information on Isotreti- noin for Females (Trade Names Being Accutane, Amnesteem, Claravis, and Sotret)}

\author{
Craig G. Burkhart, MD, MPH
}

5600 Monroe Street, Suite 106B, Sylvania, Ohio 43560, USA

There is an extremely high risk of major human fetal abnormalities if pregnancy occurs while taking isotretinoin in any amount even for short periods of time. The rules for taking isotretinoin (if female) might seem excessive, but in 2005 (before iPledge) there were over 100 females who got pregnant while on isotretinoin.

I only use isotretinoin on females if we can agree to follow all sections of the iPledge Program's "Isotretinoin Educational Kit for Female Patients Who Can Get Pregnant." I will not budge from any of the requirements listed in the booklet. Moreover, in the present legal environment in Ohio and Michigan, I can not accept abstinence as a viable method of birth control for my patients.

I require the iPledge Program "Contraception Referral Form and Contraception Counseling Guide" to be filled out by your ob/gynecologist or family doctor prior to starting accutane, as discussed on page 6 of your booklet in this designated section of your booklet. The forms of birth control which you select must be documented as you will have to enter the exact same information as Dr. Burkhart into separate computer entries to iPledge before receiving the medication from the pharmacy. My office has to register you into the iPledge system one month prior to obtaining the first prescription. One must have one negative pregnancy test prior to this registration process. Thereafter, iPledge requires additional pregnancy blood tests 1 to 3 days prior to each monthly office visit to the office, as well as for two additional months after one's last treatment.

One must use 2 forms of effective contraception for at least 1 month prior to initiation of isotretinoin therapy, during isotretinoin therapy, and for 1 month after discontinuing isotretinion therapy. One must realize that counseling about contraception and behaviors associated with an increased risk of pregnancy must be repeated on a monthly basis. Any patient can call me at any time if questions on this arise during the duration of treatment and/or 1 month after its completion. One must wait three months after discontinuing treatment before considering getting pregnant.

The DVD mentioned in the booklet is available in Dr. Burkhart's office, but will only be given out if requested. The DVD merely goes over the kinds of birth defects that can happen if one does become pregnant while on isotretinoin. The iPledge Program Checklist on page 5 of your booklet and the patient flowchart at the end of your booklet are excellent overviews of the chores that must be performed to use this drug. All the consent forms required to be completed are in your booklet.

After you have signed the consent forms and have performed the initial pregnancy test, my office will register you into the program. In about three weeks, the iPledge Program will mail you information (including an initial password) that you will need after your next visit to our office. In short, on your next visit before starting Accutane, you must (1) get a second pregnancy test a day or two before that visit; (2) get the initial prescription for Accutane during that office visit and have us enter the required info back to iPledge; (3) log into www.ipledgeprogram.com (or call 1-866-495-0654) with the provided temporary password, reset with a new password, enter in a Date of Personal Significance (date of birth, etc.), then proceed to answer all other necessary queries; and (4) proceed to the pharmacy to obtain the drug. Note: It is possible that you might have to call 1866-495-0654 to obtain a password if one has not been sent to you via U.S. mail or email.

\section{REFERENCES}

[1] Burkhart CN, Burkhart CG. Antibiotic resistant Propionibacteria acnes may not be the major issue clinically or microbiologically in acne. Br J Dermatol 2003; 148: 365-7.

[2] Burkhart CN, Burkhart CG. Microbiology's principle of biofilms as a major factor in the pathogenesis of acne vulgaris. Int J Dermatol 2003; 42: 925-7

[3] Burkhart CG. Acne biofilms, temperature, and microenvironments. J Am Acad Dermatol 2005; 52: 182.

[4] Bruggeman H, Henne A, Hoster F. The complete genome sequence of Propionibacterium acnes, a commensal of human skin. Science 2004; 305: 671-3. 
[5] Burkhart CN, Burkhart CG. Genome sequence of Propionibacterium acnes reveals immunogenic and surface-associated genes confirming existence of the acne biofilm. Int J Dermatol 2006; 45: 872.

[6] Burkhart CG, Burkhart CN. Propionibacterium acnes: Indigenous bacteria may be pathogenic in several cutaneous disease states. Arch Dermatol 2001; 137: 1250.

[7] Ramage G, Tunney MM, Patrick S, Gorman SP, Nixon JR. Formation of Propionibacterium acnes biofilms on orthopedic biomaterial and their susceptibility to antimicrobials. Biomaterials 2003; 24: 3221-7.

[8] Gao TW, Li CY, Zhao XD, Liu YF. Fatal bacteria granuloma after trauma: a new entity. Br J Dermatol 2002; 147: 985-93.

[9] Xu XL, Gao TW, Sun YQ. The study of pathogenic bacteria of fatal bacteria granuloma after trauma. Chin J Lab Med 2005; 28 : 619-21.

[10] Burkhart CN, Specht M, Neckers D. Synergistic activity of benzoyl peroxide and erythromycin. Skin Pharmacol Appl Skin Physiol 2000; 13: 292-296.

[11] Eady EA, Farmery MR, Ross JI, Cove JH, Cunliffe WJ. Effects of benzoyl peroxide and erythromycin alone and in combination against antibiotic-sensitive and -resistant skin bacteria from acne patients. Br J Dermatol 1994; 131: 331-6.

[12] Gloor M, Pfahler E, Neumann W, Hoffmann M, Schamm U. Topical treatment of acne vulgaris with erythromycin and benzoyl peroxide. Z Hautkr 1982; 57: 867-78.

[13] Eady EA, Bojar RA, Jones CE, Cove JH, Holland K, Cunliffe WJ. The effects of acne treatment with a combination of benzoyl peroxide and erythromycin on skin carriage of erythromycin-resistant Propionibacteria. Br J Dermatol 1996; 134: 107-13.

[14] Warner GT, Plosker GL. Clindamycin/benzoyl peroxide gel: a review of its use in the management of acne. Am J Clin Dermatol 2002; 3: 349-60.

[15] Ross JI, Snelling AM, Carnegie E, et al. Antibiotic-resistant acne: lessons from Europe. Br J Dermatol 2003; 148: 467-78.

[16] Gupta AK, Lynde CW, Kunynetz RA, Amin S, Choi K, Goldstein E. A randomized, double-blind, multi-center, parallel group study to compare relative efficacies of the topical gels $3 \%$ erythromycin $/ 5 \%$ benzoyl peroxide and $0.025 \%$ tretinoin/erythromycin $4 \%$ in the treatment of moderate acne vulgaris of the face. J Cutan Med Surg 2003; 7: 31-7.

[17] Burkhart CG, Burkhart CN. Treatment of acne vulgaris without antibiotics: tertiary amine-benzoyl peroxide combination $v s$ benzoyl peroxide alone (Proactiv Solution). Int J Dermatol 2007; 46: 89-93.

[18] Vazquez B, Elvira C, Roman JS. Reactivity of a polymerizable amine activator in the free radical copolymerization with methyl methacrylate and surface properties of copolymers. Polymer 1997; 38: 4365-4372.

[19] Vazquez B, Elvira C, Levenfeld B. Application of tertiary amines with reduced toxicity to the curing process of acrylic bone cements. J Biomed mater Res 1997; 34: 129-136.

[20] Moane S, Raftery DP, Smyth MR. Decomposition of peroxides by transition metal ions in anaerobic adhesive cure chemistry. Int $\mathbf{J}$ Adhesion Adhesives 1999; 19: 49-57.

[21] Vazquez B, Levenfeld B, Roman JS. Role of amine activators on the curing parameters, properties and toxicity of acrylic bone cements. Polymer Int 1998; 46: 241-250.

[22] Coates P, Vyakrnam S, Eady EA, Jones CE, Cove JH, Cunliffe WJ Prevalence of antibiotic-resistant Propionibacteria on the skin of acne patients: 10-year surveillance data and snapshot distribution study. Br J Dermatol 2002; 146: 840-8.

[23] Eady EA. Bacterial resistance in acne. Dermatology 1998; 196: 59 66.

[24] Burkhart CG, Burkhart CN. Expanding the microcomedone theory and acne therapeutics: Propionibacterium acnes biofilm produces biological glue that holds corneocytes together to form plug. J Am Acad Dermatol 2007; 57: 722-4.

[25] Wilkin J, Dahl M, Detmar M, Drake L, Feinstein A, Odom R, Powell F. Standard classification of rosacea: report of the National Rosacea Society expert committee on the classification and staging of rosacea. J Am Acad Dermatol 2002; 46: 584-7.

[26] Burkhart CG, Burkhart CN. Therapeutic implications of chemically- and biologically-altered sebum being one cause of seborrheic dermatitis. Open Dermatol J 2008; 2: 1-4 http://www.bentham.org/ open/todj/openaccess $2 . h$ tm. 